\title{
VEHICLE/PEOPLE ACCESS CONTROL SYSTEM FOR SECURITY MANAGEMENT IN PORTS
}

\author{
F. GARZIA ${ }^{1,2}$, E. SAMMARCO ${ }^{3} \&$ R. CUSANI ${ }^{1}$ \\ ${ }^{1}$ Department of Information, Electronics and Telecommunication Engineering, Sapienza, \\ University of Rome, Italy. \\ ${ }^{2}$ Wessex Institute of Technology, Southampton, UK. \\ ${ }^{3}$ General Direction of Safety, Security and Civil Protection, Vatican City State, Italy.
}

\begin{abstract}
Ports are opened infrastructures whose security is influenced by a plenty of parameters such as people/ vehicle flows. The movement of these flows must be accurately checked and controlled to ensure a correct management of security. In ports characterized by high flows, the security management must unavoidably use integrated access control systems that ensure a proper organization of the flows themselves. The present paper illustrates the work made to design and realize the integrated access control system of the most important commercial and tourist ports of Italy. Since the core of the access control system is represented by a server farm that must operate with a high efficiency, to guarantee a fast response to the high flows of people and vehicles, the system is endowed with a loading balance mechanism of server farm itself, based on genetic controller, that ensures optimal operative velocities and performances, as demonstrated in the paper.

Keywords: Access control system, genetic controller, integrated security system, security system.
\end{abstract}

\section{INTRODUCTION}

Security management in a complex context tends to be more efficient when integrated systems are used [1-3]. Ports represent complex opened infrastructures inviting great flows of people and vehicles that must be correctly controlled and managed to ensure a certain requested level of security. For this reason it is necessary to use integrated access control systems that allow the port authorities to keep security under control.

If correctly designed and integrated, access control systems allow to comply with international law prescriptions, such as International Ship and Port Facility Security (ISPS), and allow for optimizing and controlling the boarding flows, reducing the boarding time and offering, at the same time, a plenty of services such as the centralized check-in service for ship companies. Such a designed and realized system is capable of reaching opening time of the entrance gate and printing tickets in less than 5 seconds.

The entrance tickets also represent the boarding tickets of the desired ship to help people in reaching the desired ship directly without passing to the ticket office, thereby greatly reducing vehicle/people flows inside the ports and subsequently the boarding time.

The system is also interfaced with all the other subjects that generally operate in ports such as the following:

1. Ship companies;

2. Custom;

3. Police;

4. Sea guard;

5. Port operators;

6. Other specific subjects. 


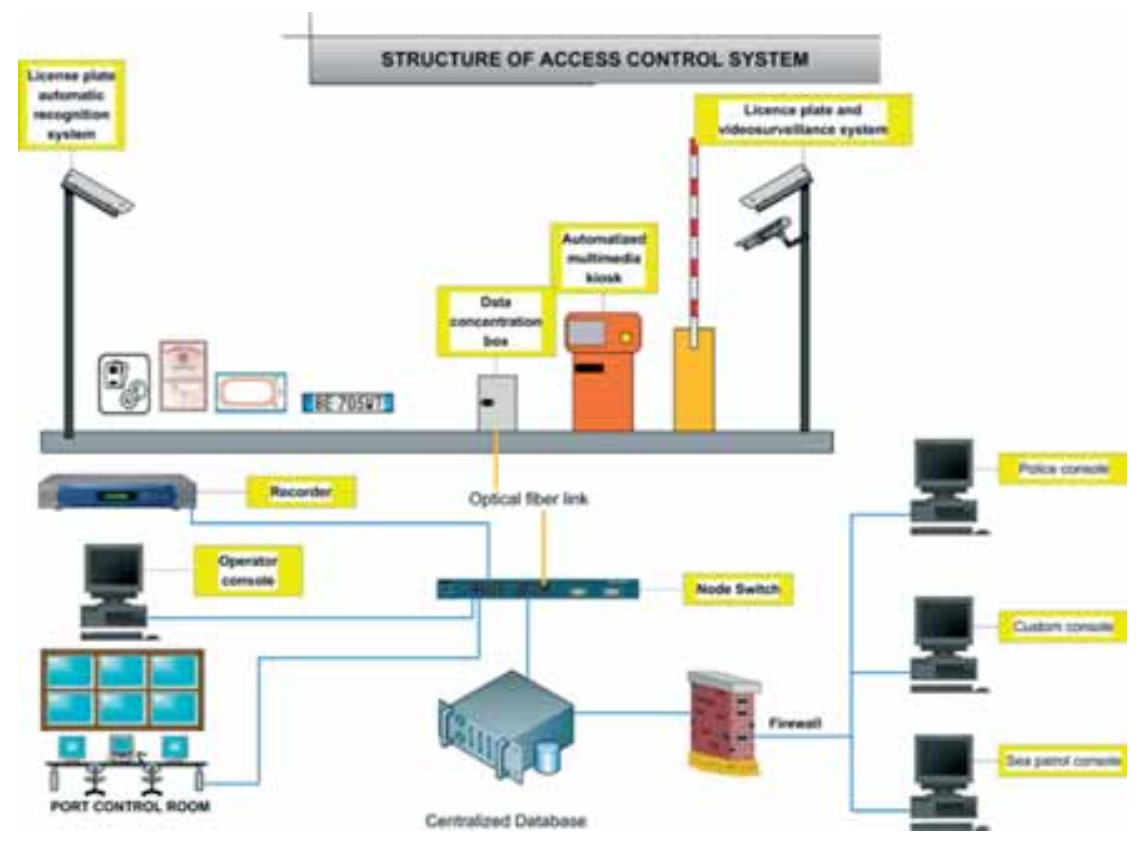

Figure 1: General scheme of the access control system.

This guarantees vehicles/people information exchange to increase the general level of security, through an easy management of information themselves.

The goal of the present paper is to illustrate a vehicle/people access control system, the difficulties found for its design and realization, and the results obtained, from its installation, in the normal and emergency situations. In Fig. 1 the general scheme of the access control system is shown.

Since the functionality of the system is ensured by a server farm, a proper genetic controller (GC) mechanism has been studied and realized. This mechanism ensures always an optimal balance of the loads of the server that compose the server farm, guaranteeing high performances of the whole system. The GC mechanism is illustrated in the following, together with the results obtained.

Due to secrecy reasons, only the general design philosophy of the vehicle/people access control system is illustrated, avoiding specific details that could compromise the security of the system itself.

\section{THE ACCESS CONTROL SYSTEM}

The system has been designed to comply with security and traffic procedures of the ports. For this reason it has been necessary to consider and respect a plenty of prescriptions and limitations imposed by the following elements:

1. ISPS code of December 2002 [ISPS is an amendment to the Safety of Life at Sea Convention (1974/1988) on minimum security arrangements for ships, ports and government agencies]. Having come into force in 2004, it prescribes responsibilities to governments, shipping companies, shipboard personnel, and port/facility personnel to 
detect security threats and take preventative measures against security incidents affecting ships or port facilities used in international trade);

2. Italian Inter-Ministerial Committee for the Maritime Safety and Security prescriptions of April 2004;

3. security evaluation document (depending on the specific port);

4. security plan of the port (depending on the specific port);

5. boarding areas and docks assignments (depending on the specific port);

6. mean boarding time of each ship company (depending on the specific port);

7. position, inside the port, of ticket offices and entrance gates (depending on the specific port);

8. position of local area network (LAN)/wide area network (optical fibre back bone) and design of the new one (depending on the specific port);

9. position of the wireless network and design of the new one (depending on the specific port);

10. network infrastructure and position of connection nodes (depending on the specific port);

11. traffic flows control video surveillance system (depending on the specific port);

12. license plate recognition system of entrance gates (to design);

13. access control multimedia kiosk endowed with automatic communication interface, radio-frequency identification device (RFID) badge reader, ticket or temporary access printer, personal identification document reader, control camera (to design).

The system allows attain the following goals:

1. entrance/exit punctual control of all the vehicles in the port area;

2. entrance/exit punctual control of all the persons in the port area;

3. access control as a function of user profile inside the port;

4. information management database available for the security services;

5. dynamic parking areas management;

6. boarding area entrances management;

7. management of the information acquired;

8. real-time data statistic of entrances, exits, parking according to different user profiles.

The entrance/exit punctual control of vehicles allows the following:

1. to have in real time all the information about the vehicles organized according to the typology or to the user profile (port worker, visitor, ship passenger, etc.);

2. the entrance of only authorized vehicles (license plate recognition system);

3. to calculate the permanence time inside the port according to different user profiles;

4. to trace the vehicles transit inside the port.

The entrance/exit punctual control of person allows

1. to have in real time all the information about the person according to the user profile (port worker, visitor, ship passenger, etc.);

2. the entrance of only authorized persons;

3. to calculate the permanence time inside the port according to different user profiles;

4. to trace the person transit inside the port.

The access control as a function of user profile inside the port allows for optimizing the entrance procedures creating proper preferential lanes inside the port according to the user profile. 
The license plate management by means of the security system allows the following:

1. real-time recognition of license plates;

2. creation of a database of undesired license plates (black list);

3. creation of database of authorized license plates (white list);

4. creation of a statistical and informative database of traffic flows.

The dynamic parking area management allows the following:

1. to optimize the parking spaces inside the port;

2. to verify in real time the entrance of users to the dedicated areas.

The real-time data statistic of entrance, exit and parking according to different user profiles allows to manage the following data:

1. entrance according to user profile;

2. exit according to user profile;

3. parking according to user profile;

4. user profile present inside the port;

and a lot of other data.

In the following sections, the different subsystems of the access control system are illustrated.

\section{TELECOMMUNICATION INFRASTRUCTURE}

The design of the system involved the design of a dedicated telecommunication system.

The telecommunication system is composed by nodes. Every node is independent and allows the connection of all the devices necessary to the access control system. In Figs. 2 and 3 the general scheme of the telecommunication system and the connection modality of the telecommunication system are respectively shown.

A proper virtual LAN is dedicated to each service inside the network, allowing to manage, in an equilibrate way, the band necessary to the communication. Nodes are connected with each other by means of optical fibers. The connections are properly made to ensure a high reliability in case of an optical fiber damage. In some places, however, it is not possible to have the optical fibers connection: in this case a broadband wireless connection was used, with a velocity equal to $300 \mathrm{Mb} / \mathrm{s}$.

All the hardware, software and network devices are placed in a proper dedicated cooled room, supplied by a proper power supplier capable of ensuring an autonomy of 24 hours in the absence of the main electrical supply.

The design of server environment has been made to make them operate in fault tolerance modality to manage high traffic peaks even in the presence of malfunctioning of one or more than one of their components.

A proper routing policy has been developed to guarantee a high security level and a high flexibility to the whole system.

For this reason different devices capable of ensuring reliability and secure communication with the different check-in and ship company clients have been integrated in the system.

The passive routing devices are properly aided by load balance devices that allow to share, in a smart way, the working duties between the different application servers. The 
loading balance of the server farm is ensured by a proper GC, as explained in the following section.

All the software has been developed using international open standards, based on more diffused framework (J2EE, MS, NET). Data storing has been made using a database management system (DBMS) relational database based on SQL standards. All the main functions of management and control are implemented on web interfaces. The web interfaces comply with normative concerning usability and accessibility. The communication and integration with other systems are based on the SOAP (XML-RPC) protocol.

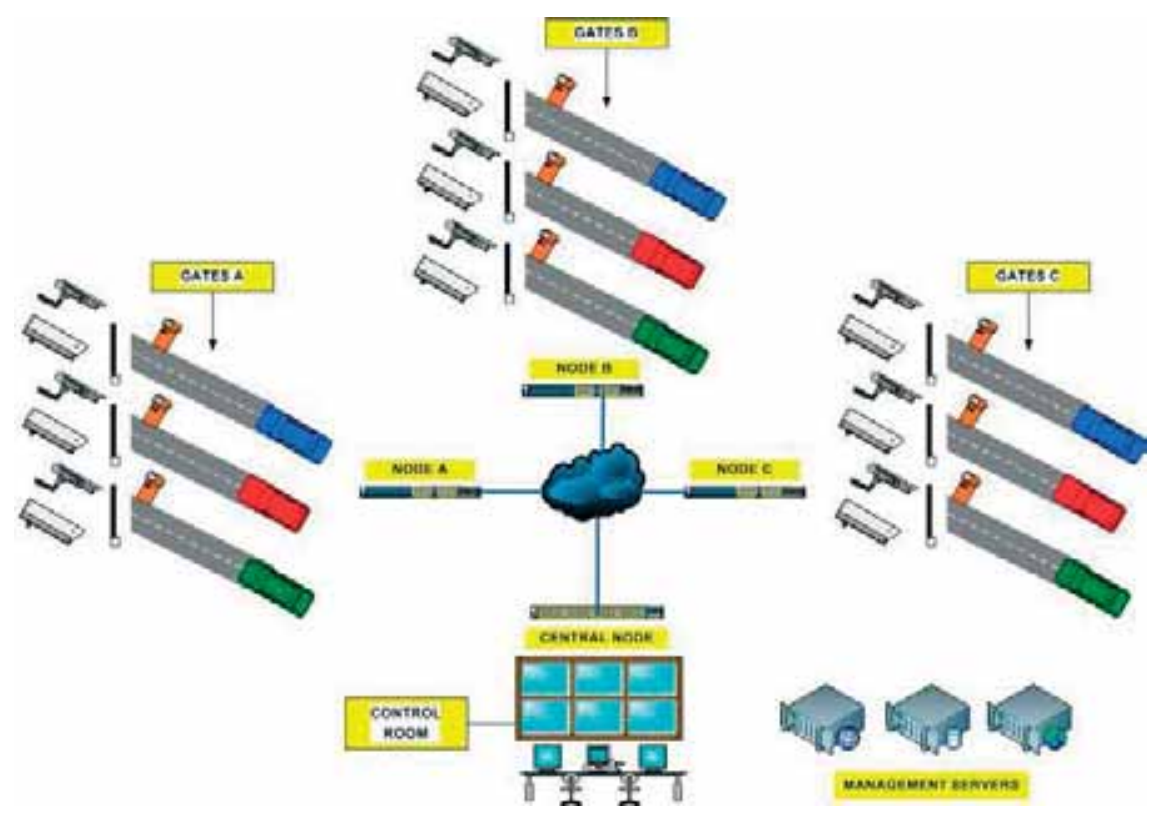

Figure 2: General scheme of the telecommunication system.

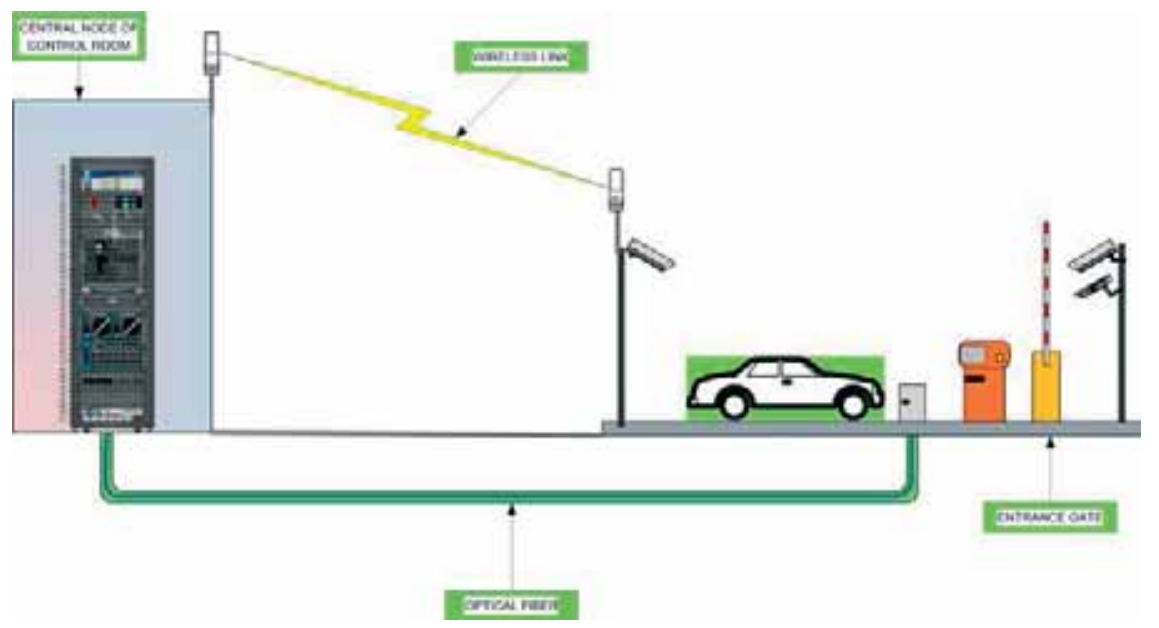

Figure 3: Connection modality of telecommunication system. 


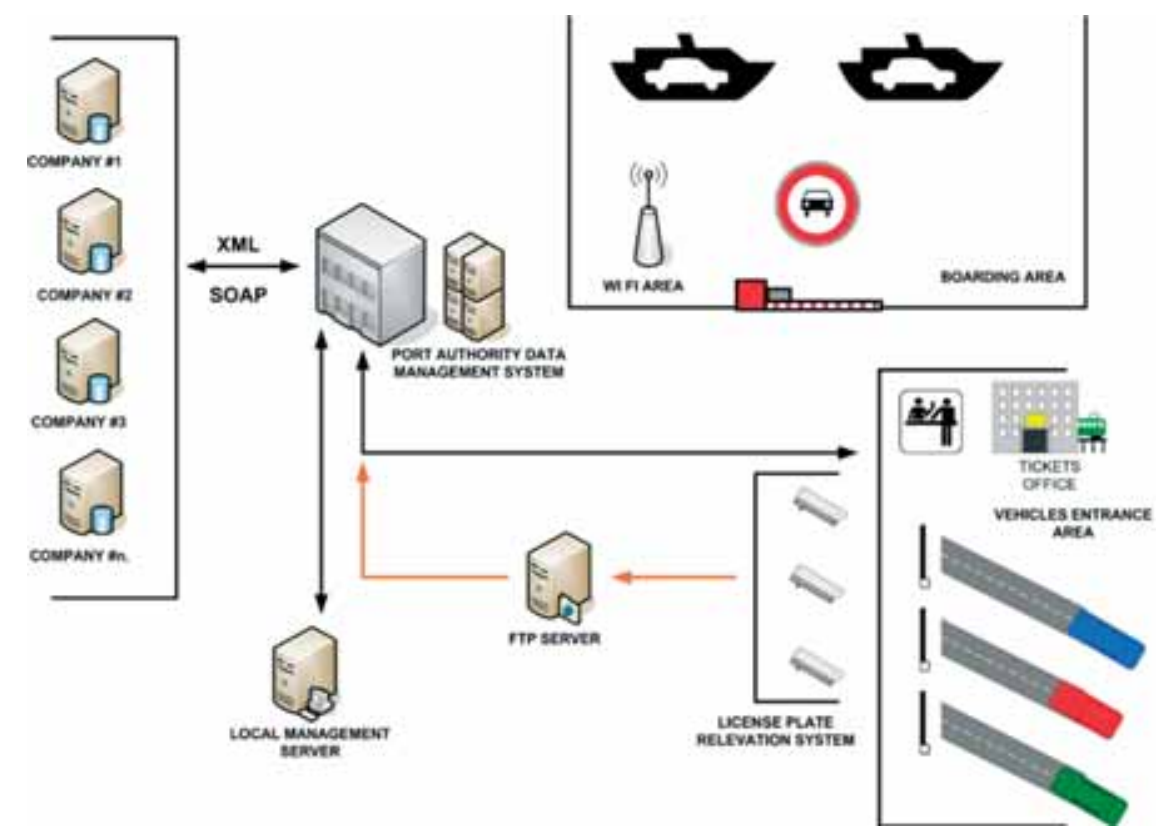

Figure 4: Scheme of the informative system.

The software architecture is based on model view controller paradigm realized by means of the design of a three-tier system that complies with W3C standard and oriented towards an approach based on service-oriented architecture services. In Fig. 4 the scheme of the informative system is shown.

The activity of installation and configuration of the whole applicative platform included the following phases:

1. realization of the central DBMS environment;

2. realization and installation of the security system integration software;

3. realization and installation of the integration software with access control system, license plate recognition system, face recognition system, RFID badge reader;

4. realization and installation of centralized check-in software;

5. design and realization of the links with the enabled port operators;

6. design and realization of the links with ship companies.

In particular, the check-in software manages and redirects the XML requests and answers of the whole system and translates them into instructions for the boarding procedures (bar opening, card printing, access control, etc.).

The centralized check-in software implements the following functionalities:

1. companies management;

2. users management;

3. multimedia kiosks management;

4. accesses control management;

5. remote configuration of entrance gates;

6. user permission management; 
7. multimedia kiosks remote assistance management;

8. ship companies XML-SOAP links management;

9. port operator external database open database connectivity link management;

10. ship companies XML link management;

11. logging access and system operation management;

12. docks and departures chart management.

Proper procedures for the integration with visual informative system of the port (public screens, etc.) have been implemented.

The system is also endowed with a proper web-based client module that ticket offices of ship companies can use to print the boarding card directly at the entrance gates.

\section{THE GATE SYSTEM}

The entrance gate is designed to use a series of sensors necessary to acquire the information dedicated to the automatic management of itself. In Fig. 5, the scheme of the entrance gate, together with sensors positioning, is shown.

Every lane uses a proper camera dedicated to the area analysis and to the image recording 24 hours a day and two further cameras dedicated to the license plates recognition (optical character recognition camera or OCR camera) that send acquired data directly to a proper multimedia kiosk.

The multimedia kiosk acquires data coming from OCR cameras and verifies the presence of the license plate in a proper white list (authorized access), black list (unauthorized access) or passenger list. According to input data, it can generate an alarm, open the entrance bar or print the check-in ticket. It is also endowed with an RFID reader that can be used by port personnel.

The gates is also equipped with two electromagnetic loops, located close to the multimedia kiosk, that avoid the bar closing when a vehicle is in the middle.

\subsection{The license plate recognition subsystem}

For license plate recognition functionality, new generation cameras have been used. They can acquire data related to high velocity traffic flows. The subsystem is placed in a proper enforced box located close to the entrance gate. In Fig. 6 an example of license plate recognition screen page of the system is shown.

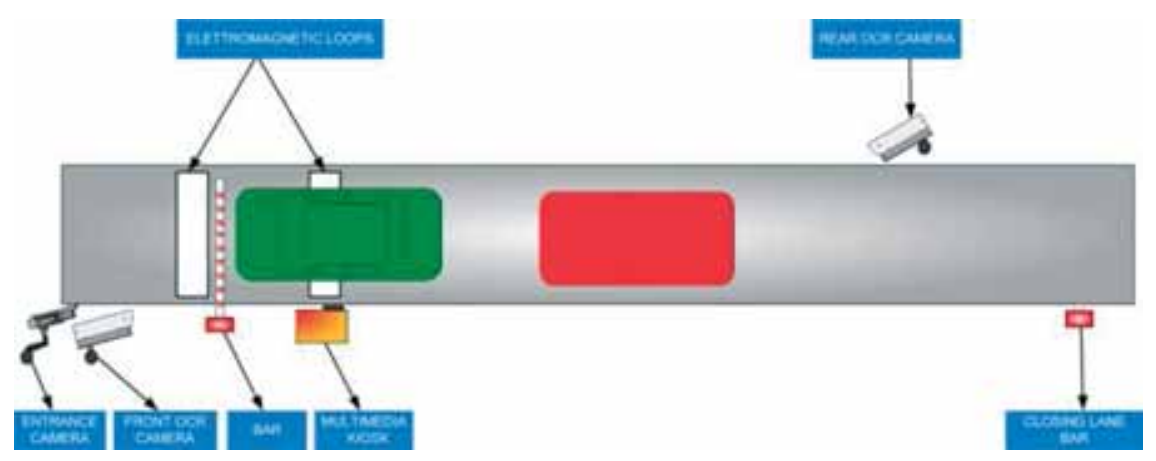

Figure 5: Scheme of the entrance gate. 


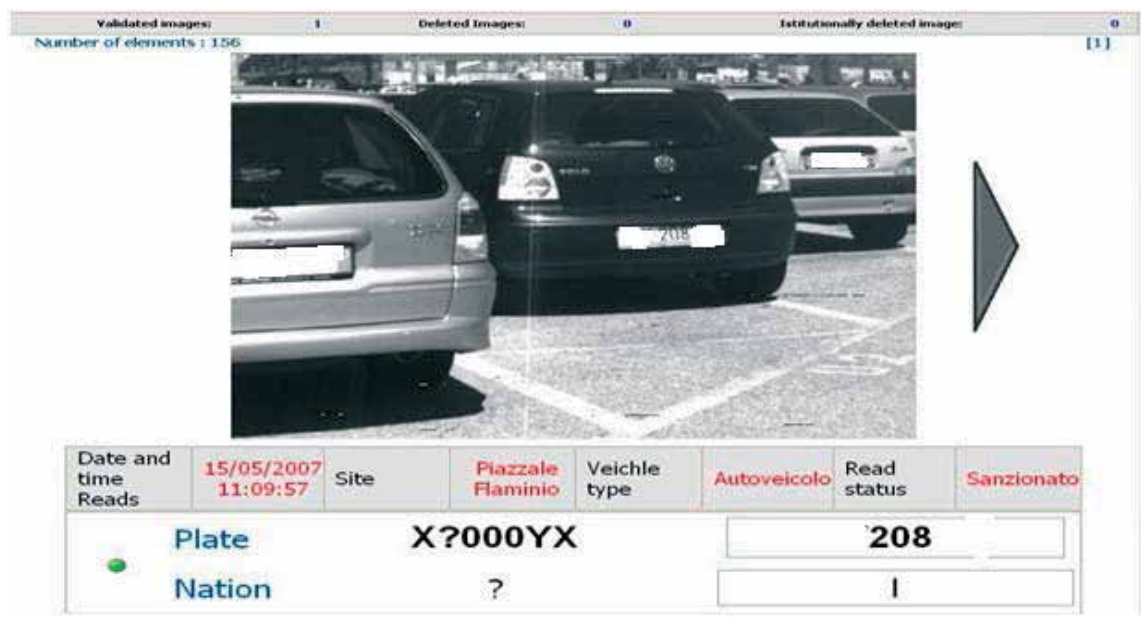

Figure 6: Example of license plate recognition screen page of the system.

The OCR cameras embed all the hardware and software necessary to the license plate recognition, ensuring a high recognition rate. They are capable of reading and recognizing the license plates present in the scene without any triggering. They are also characterized by reduced dimension that greatly simplify their installation.

The OCR camera is constituted by a binocular optics where a black/white high resolution element $(1400 \times 1024$ pixels $)$ and a color high resolution element $(1400 \times 1024$ pixels $)$ are installed. The processing unit is composed by a triple element (floating point gate array or FPGA, digital signal processing or DSP and central processing unit or CPU): the owner software, named $\mathrm{O}^{2} \mathrm{CR}$, works on the dedicated DSP unit. The OCR cameras are also equipped with infrared light to ensure a clear night vision. They communicate by means of a transmission control protocol/Internet protocol (TCP/IP) Ethernet 10/100 Mbps connection.

Thanks to the their computation capability, they are able of processing directly on board the license plates data acquired, greatly improving the performances of the whole system.

The OCR cameras divide the recognition process into three phases managed properly by the three built-in components as follows:

1. the FPGA executes a preprocessing of the images;

2. the DSP executes the real recognition of license plate;

3. the CPU executes the management and communication of license plates data.

The $\mathrm{O}^{2} \mathrm{CR}$ software can be configured to recognize, in an easy way by means of reading rules, the most of European and extra European license plates.

In case of interruption of communication network, the system stores the data related to the license plates and traffic flow of the gate and transmits them to the central database of the control room when the communication is restored.

\subsection{The video surveillance subsystem}

The entrance gates are also equipped with a videosurveillance system that allows to monitor and record image using the telecommunication network. 
The system is based on TCP/IP network protocol to allow a more flexible management of itself. In fact, if the network is properly designed, it is possible in any moment to add or remove cameras without introducing further connection cable, ensuring a high modularity of the system.

The cameras send their images to the central control room using the fixed network or the wireless network. The images are stored, in a digital way, on proper digital recorders.

The system is composed of the following:

1. IP videocameras with power over Ethernet and dome functionalities;

2. digital videorecording system;

3. management system.

\section{THE LOADING BALANCE SYSTEM OF THE SERVER FARM BASED ON GC}

Due to the high efficiency requested to the system, it is necessary to balance the computation load of the different servers that compose the server farm.

To reach this goal a genetic classifier has been used.

A genetic classifier is essentially a classifier system endowed by proper genetic algorithms (GAs) that manage its activities.

GCs have been shown to be extremely useful in a plenty of applications [4-8].

\subsection{Structure}

A classifier system is an automatic knowledge machine that is capable of learning simple rules, called classifiers, to finalize its behavior in an arbitrary environment according to determined needs.

Its structure can be described using a methodology similar to the one used for dynamic systems, that is,

1. a group of fixed length strings (classifiers), which represents the behavioral rules, based on a ternary alphabet, composed by a condition and an action. Every classifier is labeled with values that represent its strength (fitness) as a function of the results obtained operating according to the action suggested by the classifier itself;

2. a series of inputs that receive the information from the external environment and determine which classifier must be activated;

3. an auction mechanism that determines which of the activated classifier is effectively acting;

4. an accountability system that updates the values of each classifier basing on the premium received according to the decision acted;

5. a GA that is capable of introducing new set of rules substituting the older ones. The algorithm is generally activated when an input message does not correspond to any classifier already present inside the system.

\subsection{Principles}

The system can be reduced to three fundamental points that are shown in Fig. 7.

\subsubsection{Rules and messages system}

The system of rules and messages present in the classifier system is a special kind of operative system. It represents a computational scheme that uses proper rules to reach the desired 


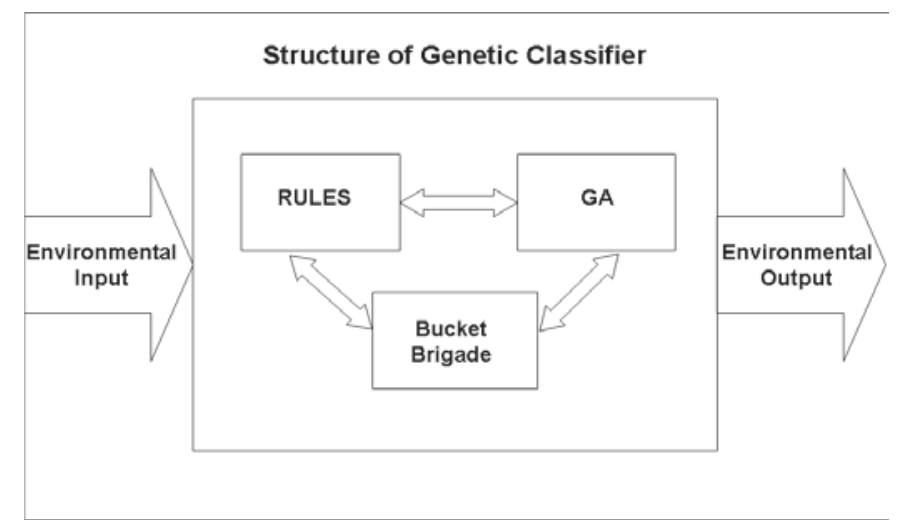

Figure 7: Scheme of a genetic classifier.

goal. It has been demonstrated [4-8] that these systems are computationally complete and efficient. Although different syntaxes are available as a function of the working scheme chosen, generally the rules can be represented as follows:

$$
\text { if }<\text { condition }>\text { then }<\text { action }>
$$

The above rules mean that the action is immediately executed if the condition is satisfied.

The classifier systems adopt a fixed length representation for the rules and allow the activation and the use of parallel rules.

The system of rules and messages constitutes the computational core of the classifier. The information propagates from the environment, through the inputs, and they are decoded into one or more than one fixed length messages. These messages can activate the related rules that are inserted in a proper list of messages.

Once a classifier is activated, it sends its message to the list above mentioned. These messages can, in a second time, activate other messages or generate an action through the actuator towards the external environment using proper effectors. In this way the classifiers combine their suggestions and the environmental suggestions to determine the future behavior of the whole system.

To understand this mechanism, it is better to analyze how the messages and the classifiers are used inside the system.

A message inside the system is simply a finite length string, composed using a finite alphabet. Since we limit, in our case, the use of a binary alphabet, its precise definitions is

$$
<\text { message }>::=\{0,1\}
$$

A message is therefore defined as a sequence of 1 and 0 and it represents the fundamental instrument for information exchange inside the whole system.

Messages inside the list can be coupled with one or more than one rules. A classifier is therefore a working rule defined as

$$
<\text { classifier }>::=<\text { condition }>:<\text { message }>
$$

where the condition is defined as

$$
<\text { condition }>::=\{0,1, \#\}
$$


It is immediate to note that the definition of a condition differs from the definition of message exclusively for the introduction of a special character (\#) that implies a 'don't care' situation. A condition is therefore coupled with a message if, in any position of its string, a 0 couple with a 0 , a 1 couple with a 1 , or a 'don't care' \# couple with a 0 or a 1.

Once a condition of the classifier is coupled, the related classifier becomes a candidate to send its message to the list of messages in the following step. The possibility of sending its message to the list is defined based on the strength of the message itself through a proper auction involving all the activated rules.

For this reason it is necessary to introduce a credit assignment algorithm that allows each classifier to be labeled with a proper strength value.

\subsubsection{Figure of merit assignment}

The most used algorithm for this kind of functionality is the so called bucket brigade. To better understand its behavior, a metaphor has been used using two main components that are an auction and a clearing house.

When the classifiers are coupled, they do not send immediately their message to the list but they participate to an auction. Each message can participate to the auction thanks to its strength that represents a concept similar to the fitness in the GA, which is the goodness of its property in solving the defined problem. Every classifier makes a bid B proportional to its strength, that is

$$
\mathrm{Bi}=\mathrm{Cbid} * \mathrm{Si}
$$

where $\mathrm{Bi}$ is the bid, $\mathrm{Si}$ is the strength of the classifier and Cbid is a proper proportionality constant.

In this way, the rules characterized by greater fitness values are classifiers to be selected to send its message. The auction allows a particular rule that has been selected through the auction to delete its bid by means of the clearing house in the case of a remainder coupling with the remaining messages.

The payment of the bid is divided between the classifiers that couple in different ways; this payoff division helps the whole system to guarantee the formation of a correctly dimensioned messages subpopulation.

\subsubsection{GA}

GA is the third and fundamental point of a classifier system.

The GA used inside the proposed genetic classifier system is operative according the following steps:

1. code of the problem;

2. creation of the initial population of potential solutions;

3. creation of a fitness function that allows each solution to be assigned with a value that estimates its suitability;

4. formalization of genetic operators (crossover, mutation, etc.) that alters the next generation chromosomes;

5. assignment of values to the different parameters that regulate the evolution (population dimension, probability of application of genetic operators);

6. definition of stop condition. 


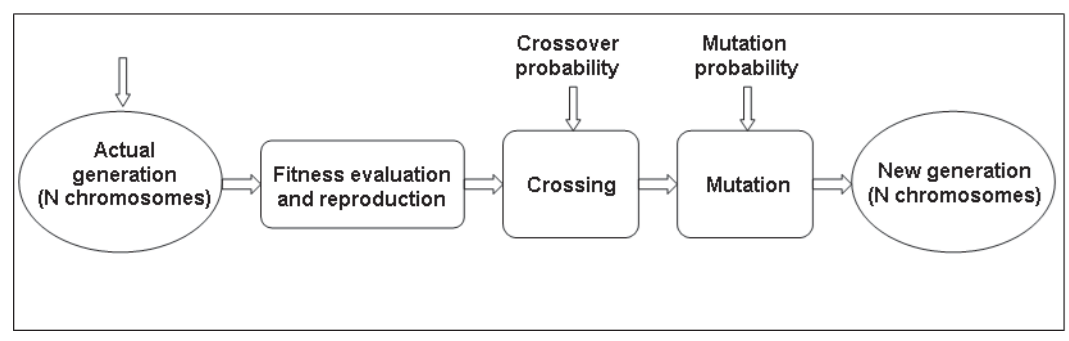

Figure 8: Operative scheme of genetic algorithms.

In our case, the GA tries to evolve, learning new rules of the kind 'if <condition> then $<$ action>' that allows the genetic classifier to operate in the better way inside the considered system.

The fitness of the rules is evaluated considering their performances in terms of correctness of load balance prediction. In this way, the GA introduces new sets of rules inside the system, deleting the older one, allowing the classifier of reaching in the better way its regime condition. The used fitness function is illustrated in the following paragraph.

The algorithm stops according to the chosen stopping conditions. Even in this case, no predefined conditions exist, and a stop condition related to the degree of fitness reached has been chosen.

The selection process is implemented by means of the so-called roulette wheel selection where the value of the strength $S$ of every classifier represents its fitness score. In Fig. 8 the operative scheme of genetic algorithms is shown.

\subsection{The proposed genetic system}

We already said that a proper protocol based on genetic classifier has been developed to optimize the load of the server farm.

In the following, the features of the considered protocol are illustrated.

Since the GC must decide which server must assume the next computation load as a function of the date, of the time and of the load of the other servers of the server farm, the structure of the rules is

$$
\begin{aligned}
& \text { If }<\text { date, time, server }{ }_{1} \text {, } \text { server }_{2}, \ldots \ldots ; \text { server }_{\mathrm{N}-1}, \text { server }_{\mathrm{N}}>\text { then } \\
& <\text { number of server to be loaded }>
\end{aligned}
$$

where $\mathrm{N}$ is the number of servers that composes the server farm.

The date and the time are inserted in the input information to consider the variability of the traffic load as a function of the different vehicle traffic of the year.

The information coming from the system is stored as environmental messages that determine which classifier must be activated based on the result of matching operation between strings of bits.

At the same time different rules can match their condition with the description of information coming from the environment: in this case, a proper auction mechanism is activated to select the most fitting classifier.

The selected rules pay a certain fee proportional to its patrimony, that is divided between the classifier that has activated it, increasing their values.

The strength of a group of rules is evaluated considering the performance of the them in terms of prediction of server load. In this way, the GA introduces new set of rules inside the 
system, substituting the older one, allowing the GC to reach its better performance in a short period.

The result of this working principle is a numerical value that represents the number of servers that must execute the next computation load, allowing the choice of the more correct one to ensure a proper load balance of the server farm, avoiding any overload of any server that would unavoidably slow the response time of the whole system.

After a certain time, depending on the variability of input requests, the GC starts to work correctly, giving to the system a high reliability and efficiency, which is one the purpose of the present work.

We want now to describe the used fitness function. During the auction process, one or more rules can participate in it. Each time a rule participates to a specific process of estimation of the server to be loaded, it is properly labeled with the number of process: one rule can participate in different processes and this information is stored in a proper label field of the rule. Once a server to be loaded is requested to the system, the system checks continuously the real duration of the load, to use this information, in a second time during the GA phase, to select the most precise rules. When the GA phase is activated, every rules is properly assigned to a portion of the roulette wheel according to its precision in the loading balance it participated, to be eventually selected for the next generation population: the more precise the forecast of the rules and the higher the occupation space in the roulette wheel and consequently the higher the possibility to be selected for the next generation of population. The fitness of each rule is calculated in the following way: for every rule a proper check about the estimation processes it participated in is made and the most precise forecast is selected, that is the rule is associated to the estimation process that differs, as less as possible, from the rules forecast. The fitness values of the rules are chosen to be variable between 10 (exact forecast) and 1 (totally wrong forecast). The value 1 has been chosen to allow also the wrong rules to evolve towards more fitting rules. This choice is very useful in the initial phase of the node, when only a few rules are used in the auction mechanism while all the other are momentarily in stand-by: since these last rules can be useful in the following phases, they must be characterized by a certain probability to pass to the next generation population of rules. If $\mathrm{L}_{\mathrm{i}}$ is the real load (in percentage) of the server $i$ and $\mathrm{L}_{\mathrm{M}}$ is mean load of the server farm, calculated as

$$
\mathrm{L}_{\mathrm{M}}=\left(\sum_{\mathrm{i}=1} \rightarrow_{\mathrm{N}} \mathrm{L}_{\mathrm{i}}\right) / \mathrm{N}
$$

the fitness value $F_{j}$ of the rules $j$ is calculated as

$$
\mathrm{F}_{\mathrm{j}}=10-\left(\mathrm{E}_{\mathrm{L}} / \mathrm{NL}_{\mathrm{M}}\right) * 10
$$

where $E_{L}$ is the sum of the differences between each server load and the mean load of the server farm, defined as

$$
\mathrm{E}_{\mathrm{L}}=\left(\sum_{\mathrm{i}=1} \rightarrow_{\mathrm{N}}\left(\mathrm{L}_{\mathrm{i}}-\mathrm{L}_{\mathrm{M}}\right)\right.
$$

It is evident that if forecasted load of rule $\mathrm{j}$ for the fitness produces a uniform load of the server farm, the fitness value $F_{j}$ of the rules $j$ is equal to 10 . If $F_{j}$ is lesser than 1 , it is set by default equal to 1 , to ensure a certain residual probability of the rule $\mathrm{j}$ to evolve in the next generation population of rules.

If a certain rule did not participate in any auction process, it is automatically rated with value 1 . If a certain rule participated in one or more different auction processes but it is rated with a value lesser than 1 , the fitness value is set to 1 .

Once all the rules are properly rated, they are assigned a space proportional to their fitness value on a proper roulette wheel and the next population selection mechanism takes place. 
The choice of the number of rules is quite critical since a reduced number (100 for example) ensures a rapid learning but a higher percentage of error while a great number (1000 for example) ensures a reduced percentage of error but a long learning time. The optimal number of classifiers in our case has resulted to be 600 , as explained in next paragraph.

In the next paragraph the results obtained from the implementation of the considered protocol are illustrated.

\subsection{Performances and results}

It is first of all necessary to define the minimum number of rules to obtain the minimum value of $E_{L}$, defined by eq. (9) as the sum of the differences between each server load and the mean load of the server farm.

The behavior of $E_{L}$ as a function of the number of rules $N_{R}$ is shown in Fig. 9.

From Fig. 9 it is possible to see that $E_{L}$ decreases as $N_{R}$, reaching an asymptotic value of about $2 \%$ when it is greater than 600 . This allows us to consider a number of rules $N_{R}$ of 600 a good compromise between precision and computation load of GC.

Once the optimal number of rules is individuated, it is necessary to consider the time necessary to reach the minimum value of $2 \%$ for $\mathrm{E}_{\mathrm{L}}$, that is, to reach an optimal loading balance of the server farm.

The behavior of $\mathrm{E}_{\mathrm{L}}$ as a function of the training time, expressed in months, is shown in Fig. 10.

From Fig. 10 it is possible to see that the GC reaches it optimal performance (minimum value of $\mathrm{E}_{\mathrm{L}}$ ) after 12 months, that is one year. This period allows the system to learn the behavior of the vehicles traffic during a whole year and to learn the correct strategy to manage

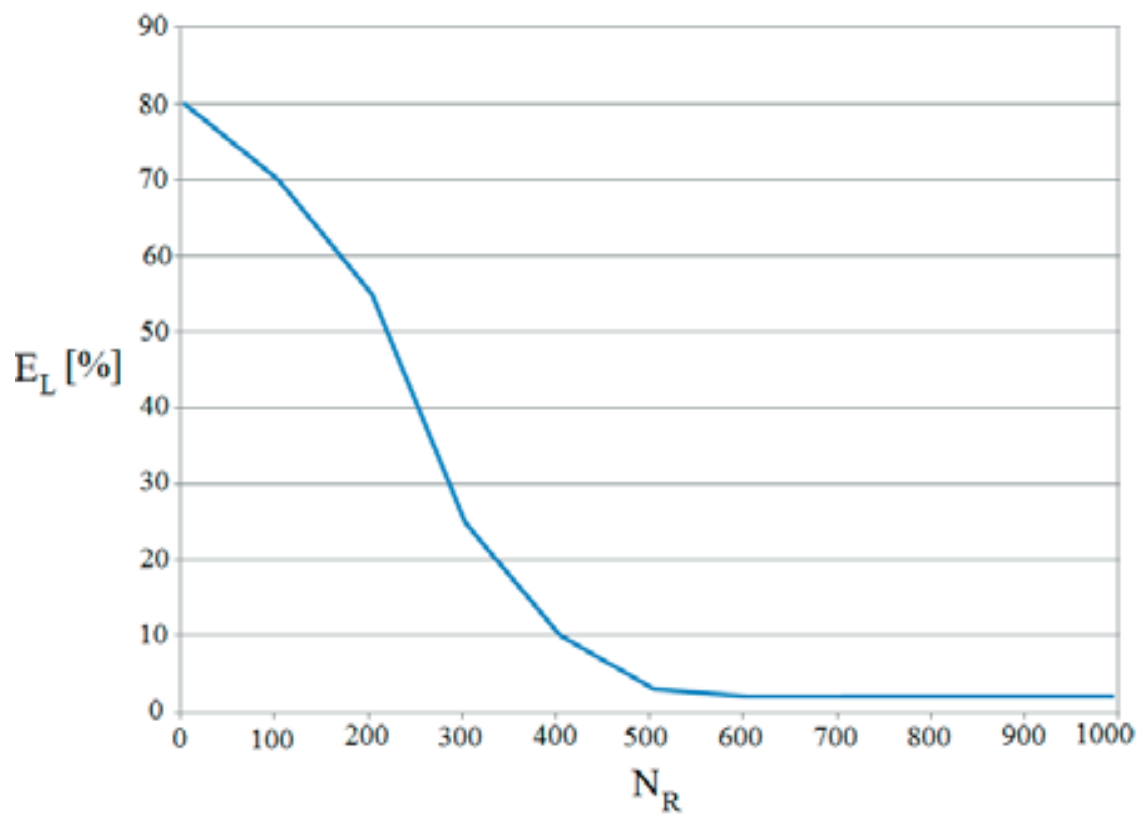

Figure 9: $E_{L}[\%]$ as a function of number of rules $N_{R}$. 


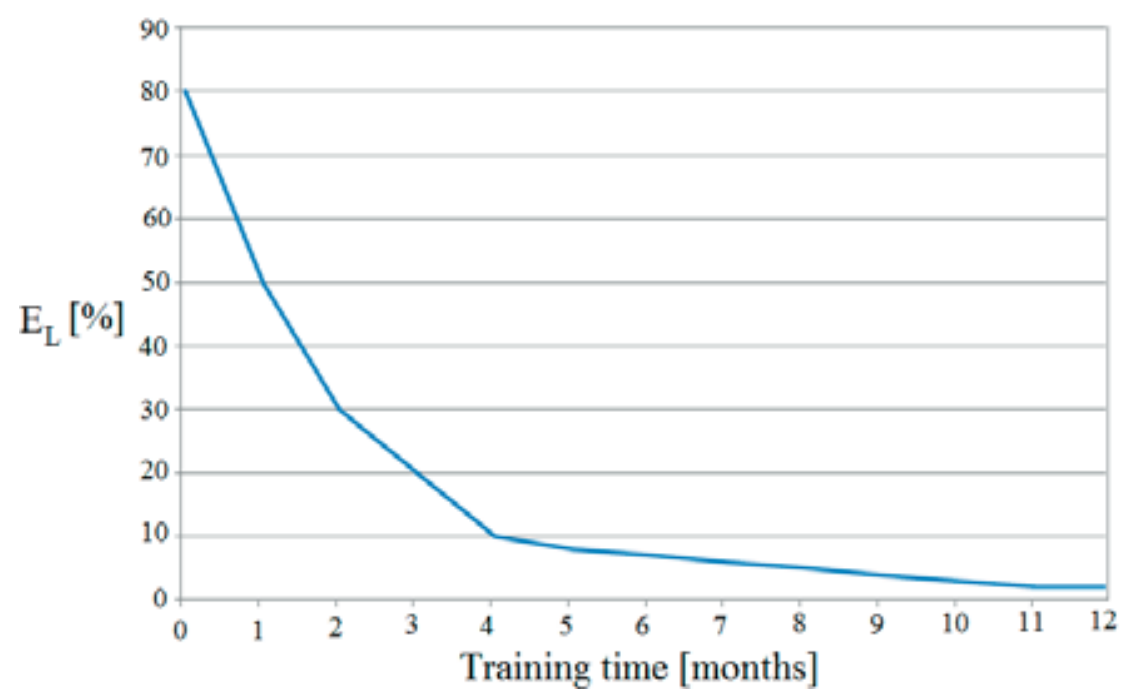

Figure 10: $\mathrm{E}_{\mathrm{L}}[\%]$ as a function of training time (months).

the server farm to obtain an optimal balance of the server farm and therefor optimal performances of the whole system.

\section{FURTHER FUNCTIONALITIES OF THE ACCESS CONTROL SYSTEM}

The system ensures further functionalities that are illustrated in the following:

First of all it allows the communication between different ports so that the entrance data of the leaving port are sent to the arrival port to be used as exit data for people and vehicles, as shown in Fig. 11.

The system prints tickets directly for passengers and vehicles with the graphic layout of the required ship company, adding a proper bar code with all the necessary information.

The tickets also contain the data related to the vehicle (height and length) acquired by means of proper sensors installed at the entrance gates: these data are very useful to the ship companies to optimize the boarding operations.

The tickets are also endowed with a proper magnetic strip that allows them to be used as key to the cabin when they are on-board the ships.

The OCR cameras are capable of recognizing the nationality of vehicles and producing directly vocal messages in the desired language by means of the multimedia kiosks of the entrance gates.

The use of wireless networks allows the use of mobile devices that ensures a series of advanced services, which is very useful for port management. In fact, all the individuals are equipped with proper wireless terminals that allow them to manage and control all the access data from any place of the port. The ship company operators can read the bar code on the tickets directly with their portable terminals and immediately acquire all the boarding information related to passenger and vehicles, reducing the boarding operations and the related time. The security personnel is equipped with proper wireless OCR cameras that read the license plates during their patrolling operations inside the port and provide them all the data related to the user profile (authorized or unauthorized vehicle, time and data of entrance, parking time, etc.). 


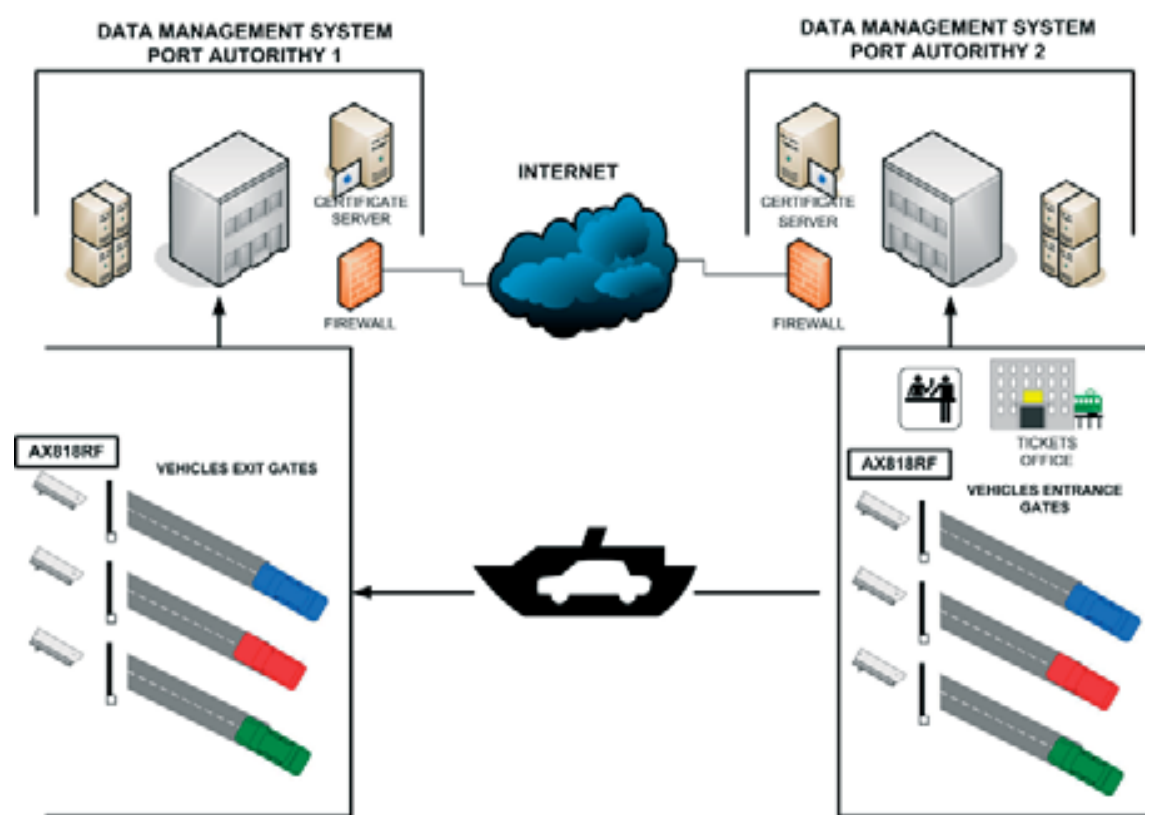

Figure 11: Scheme of the interconnection architecture between different ports.

\section{OPERATIVE RESULTS}

A proper reliability analysis was made on the system to individuate the most critical components and to make a proper predictive maintenance, avoiding malfunctioning of the system. This has always ensured a full functionality and availability of the system.

Slight modifications were made on the software to modify the system according to the variations requested by the different subjects that use the system (Ship Companies, Custom, Police, Sea Guard, Port Operators, etc.).

All the users have been fully satisfied by the performances of the system and by its possibility to be modified as a function on the different variations that took place after its starting, ensuring a high adaptability to the operative scenario.

\section{CONCLUSIONS}

The illustrated access control system has been realized in the main ports of Italy and it has controlled, for different years, millions of vehicle/people per year, showing a great efficiency in security management [9].

Thanks to the GC-based mechanism of the load management of the server farm, the system has been able to work always at its maximum velocity and performances, never showing any overload or any slowing of its response time.

\section{REFERENCES}

[1] Garzia, F., Sammarco, E. \& Cusani, R., The integrated security system of the Vatican City State. International Journal of Safety \& Security Engineering, 1(1), pp. 1-17, 2011, doi: 10.2495/SAFE-V1-N1-1-17. doi: http://dx.doi.org/10.2495/SAFE-V1-N1-1-17 
[2] Contardi, G., Garzia, F. \& Cusani, R., The integrated security system of the Senate of the Italian Republic. International Journal of Safety \& Security Engineering, 1(3), pp. 219-247, 2011. doi: http://dx.doi.org/10.2495/SAFE-V1-N3-219-247

[3] Garzia, F. \& Cusani, R., The safety/security/communication system of the Gran Sasso mountain in Italy. In print on International Journal of Safety \& Security Engineering.

[4] McAulay, A.D. \& Chan Oh, J. Improving learning of genetic rule-based classifier system. IEEE Transactions On Systems, Man, And Cybernetics, 24(I), pp. 152-159, 1994. doi: http://dx.doi.org/10.1109/21.259696

[5] Pozo, A.R. \& Hasse, M., “A genetic classifier tool”, Computer Science Society, 2000. SCCC '00. Proceedings. XX International Conference of the Chilean, pp. 14-23, 2000. doi: http://dx.doi.org/10.1109/SCCC.2000.890387

[6] Castillo, C., Lurgi, M. \& Martinez, I., "Chimps: an evolutionary reinforcement learning approach for soccer agents", Systems, Man and Cybernetics, 2003. IEEE International Conference On, 1, pp. 60-65, 2003.

[7] Liu, B., McKay, B. \& Abbass, H.A. "Improving genetic classifiers with a boosting algorithm”, Evolutionary Computation, 2003. CEC '03. The 2003 Congress On, 4, pp. 2596-2602, 2003.

[8] Garzia, F., Perna, C. \& Cusani, R. Ad Hoc network hybrid management protocol based on genetic classifiers. Int. J. Wireless Engineering and Technology, 1(2), pp. 69-80, 2010. doi: http://dx.doi.org/10.4236/wet.2010.12011

[9] Web site of the Association of Italian ports: www.assoporti.it 